\title{
2413-balloon permutations and the growth of the Möbius function
}

\author{
David Marchant \\ School of Mathematics and Statistics \\ The Open University \\ Milton Keynes, MK7 6AA, UK \\ david.marchant@open.ac.uk
}

Submitted: Mar 5, 2019; Accepted: Dec 9, 2019; Published: Jan 10, 2020

(C) The author. Released under the CC BY-ND license (International 4.0).

\begin{abstract}
We show that the growth of the principal Möbius function on the permutation poset is at least exponential. This improves on previous work, which has shown that the growth is at least polynomial.

We define a method of constructing a permutation from a smaller permutation which we call "ballooning". We show that if $\beta$ is a 2413-balloon, and $\pi$ is the 2413balloon of $\beta$, then $\mu[1, \pi]=2 \mu[1, \beta]$. This allows us to construct a sequence of permutations $\pi_{1}, \pi_{2}, \pi_{3} \ldots$ with lengths $n, n+4, n+8, \ldots$ such that $\mu\left[1, \pi_{i+1}\right]=2 \mu\left[1, \pi_{i}\right]$, and this gives us exponential growth. Further, our construction method gives permutations that lie within a hereditary class with finitely many simple permutations.

We also find an expression for the value of $\mu[1, \pi]$, where $\pi$ is a 2413-balloon, with no restriction on the permutation being ballooned.
\end{abstract}

Mathematics Subject Classifications: 05A05

\section{Introduction}

Let $\sigma$ and $\pi$ be permutations of natural numbers, written in one-line notation, with $\sigma=\sigma_{1} \sigma_{2} \ldots \sigma_{m}$, and $\pi=\pi_{1} \pi_{2} \ldots \pi_{n}$. We say that $\sigma$ is contained in $\pi$ if there is a sequence $1 \leqslant i_{1}<i_{2}<\ldots<i_{m} \leqslant n$ such that for any $r, s \in\{1, \ldots, m\}, \pi_{i_{r}}<\pi_{i_{s}}$ if and only if $\sigma_{r}<\sigma_{s}$. We say that $\pi$ avoids $\sigma$ if $\pi$ does not contain $\sigma$. The set of all permutations is a poset under the partial order given by containment.

A closed interval $[\sigma, \pi]$ in a poset is the sub-poset $\{\tau: \sigma \leqslant \tau \leqslant \pi\}$, and a half-open interval $[\sigma, \pi)$ is the sub-poset $\{\tau: \sigma \leqslant \tau<\pi\}$. The Möbius function $\mu[\sigma, \pi]$ is defined 
on an interval of a poset as follows: for $\sigma \not \leq \pi, \mu[\sigma, \pi]=0$; for all $\pi, \mu[\pi, \pi]=1$; and for $\sigma<\pi$

$$
\mu[\sigma, \pi]=-\sum_{\lambda \in[\sigma, \pi)} \mu[\sigma, \lambda]
$$

In this paper we are principally concerned with the growth of the principal Möbius function, $\mu[\pi]=\mu[1, \pi]$.

Applying the Möbius function to the permutation poset was first mentioned by Wilf [8]. Burstein, Jelínek, Jelínková and Steingrímsson [4] ask whether the principal Möbius function is unbounded, which is the first reference to the growth of the Möbius function in the literature. They show that $\mu[\pi] \in\{0, \pm 1\}$, and thus is bounded, if $\pi$ is a separable permutation, and so is in a hereditary class with simples $\{1,12,21\}$. They ask (Question 27 ) for which classes is $\mu[\pi]$ bounded?

Smith [6] found an explicit formula for the principal Möbius function for all permutations with a single descent. This shows that the growth of the Möbius function is at least quadratic. Jelínek, Kantor, Kynčl and Tancer [5] show how to construct a sequence of permutations where the absolute value of the Möbius function grows according to the seventh power of the length. In the other direction, Brignall, Jelínek, Kynčl and Marchant [2] show that the proportion of permutations of length $n$ with principal Möbius function equal to zero is asymptotically bounded below by $(1-1 / e)^{2} \geqslant 0.3995$.

We show that, given some permutation $\beta$, we can construct a permutation that we call the "2413-balloon" of $\beta$. This permutation will have four more points than $\beta$. We then show that if $\pi$ is a 2413-balloon of $\beta$, and $\beta$ is itself a 2413-balloon, then $\mu[\pi]=2 \mu[\beta]$. From this we deduce that the growth of the principal Möbius function is exponential. If $\beta=25314$ (which is a 2413-balloon), then we can construct a hereditary class that contains only the simple permutations $\{1,12,21,2413,25314\}$, where the growth of the principal Möbius function is exponential, answering questions in Burstein et al [4] and Jelínek et al [5].

We start by recalling some essential definitions and notation in Section 2, where we also provide some extensions of existing results. We formally define a 2413-balloon in Section 3, and we provide some results which will be used in the remainder of this paper. In Section 4, we derive an expression for the value of $\mu[\pi]$ when $\pi$ is a double 2413balloon, and following this we show that the growth of the Möbius function is exponential in Section 5. We return to the topic of 2413-balloons in Section 6, and derive an expression for the value of $\mu[\pi]$ when $\pi$ is any 2413-balloon. Finally, we discuss the generalization of the balloon operator in Section 7. We also ask some questions regarding the growth of the Möbius function. 


\section{Essential definitions, notation, and results}

In this section we recall some standard definitions and notation that we will use, and add some simple definitions and consequences of known results.

An interval in a permutation $\pi$ is a contiguous set of indexes $i, i+1, \ldots, j$ such that the set of values $\left\{\pi_{i}, \pi_{i+1}, \ldots, \pi_{j}\right\}$ is also contiguous. Every permutation $\pi$ has intervals of length 1 and of length $|\pi|$, which we call trivial intervals. A simple permutation is a permutation that only has trivial intervals. As examples, 1324 is not simple, as, for example, the second and third points (32) form a non-trivial interval, whereas 2413 is simple.

Given two permutations $\alpha$ and $\beta$, with lengths $a$ and $b$ respectively, the direct sum of $\alpha$ and $\beta$, written $\alpha \oplus \beta$ is the permutation $\alpha_{1}, \ldots, \alpha_{a}, \beta_{1}+a, \ldots, \beta_{b}+a$. The skew sum, $\alpha \ominus \beta$, is the permutation $\alpha_{1}+b, \ldots, \alpha_{a}+b, \beta_{1}, \ldots, \beta_{b}$.

Let $\alpha$ be a permutation, and $r$ a positive integer. Then $\oplus^{r} \alpha$ is $\alpha \oplus \alpha \oplus \ldots \oplus \alpha \oplus \alpha$, with $r$ occurrences of $\alpha$.

If $\pi$ is a permutation with length $n$, then the number of corners of $\pi$ is the number of points of $\pi$ that are extremal in both position and value, that is, $\pi_{1} \in\{1, n\}$ or $\pi_{n} \in\{1, n\}$. It is easy to see that any permutation with length 2 or more can have at most two corners. We adopt the convention that the permutation 1 has one corner.

If a permutation $\pi$ can be written as $1 \oplus 1 \oplus \tau, 1 \ominus 1 \ominus \tau, \tau \oplus 1 \oplus 1$, or $\tau \ominus 1 \ominus 1$, where $\tau$ is non-empty (so $|\pi| \geqslant 3$ ), then we say that $\pi$ has a long corner.

We now have

Lemma 1. If $\pi$ has a long corner, then $\mu[\pi]=0$.

Lemma 2. If $\pi$ can be written as $\pi=1 \oplus \tau$, or $\pi=\tau \oplus 1$ or $\pi=1 \ominus \tau$ or $\pi=\tau \ominus 1$, and does not have a long corner, then $\mu[\pi]=-\mu[\tau]$.

These are well-known consequences of Propositions 1 and 2 of Burstein, Jelínek, Jelínková and Steingrímsson [4], and we refrain from providing proofs here. The reader is directed to Lemma 4 in [3] for a proof of Lemma 1. Lemma 2 is a trivial extension of Corollary 3 in $[4]$.

A triple adjacency is a monotonic interval of length 3. Smith shows that

Lemma 3 (Smith [6, Lemma 1]). If a permutation $\pi$ contains a triple adjacency then $\mu[\pi]=0$.

A trivial corollary to Lemma 3 is

Corollary 4. If a permutation contains a monotonic interval with length 3 or more, then $\mu[\pi]=0$. 
A chain in a poset interval $[1, \pi]$ is, for our purposes, a subset of the permutations in the interval $[1, \pi]$, where the subset includes the elements 1 and $\pi$, and any two elements of the subset are comparable. This last clause means that the subset has a total order. If a chain $c$ has $C$ elements, then we say that the length of $c$, written $|c|$, is $C-1$.

Philip Hall's Theorem[7, Proposition 3.8.5] says that

$$
\mu[\sigma, \pi]=\sum_{c \in \mathcal{C}(\sigma, \pi)}(-1)^{|c|}=\sum_{i=1}^{|\pi|-1}(-1)^{i} K_{i}
$$

where $\mathcal{C}(\sigma, \pi)$ is the set of chains in the poset interval $[\sigma, \pi]$ which contain both $\sigma$ and $\pi$, and $K_{i}$ is the number of chains of length $i$.

If $\mathcal{C}$ is a subset of the chains in some poset interval $[\sigma, \pi]$, then the Hall sum of $\mathcal{C}$ is $\sum_{c \in \mathcal{C}}(-1)^{|c|}$.

A parity-reversing involution, $\Phi: \mathcal{C} \mapsto \mathcal{C}$, is an involution such that for any $c \in \mathcal{C}$, the parities of $c$ and $\Phi(c)$ are different.

A simple corollary to Hall's Theorem is

Corollary 5. If we can find a set of chains $\mathcal{C}$ with a parity-reversing involution, then the Hall sum of $\mathcal{C}$ is zero.

Proof. Because there is a parity-reversing involution, the number of chains in $\mathcal{C}$ with odd length is equal to the number of chains with even length, so $\sum_{c \in \mathcal{C}}(-1)^{|c|}=0$.

We can also use Hall's Theorem if we have a subset of chains that meet a specific criteria:

Lemma 6. Let $\pi$ be any permutation with length three or more. Let $\psi$ be a permutation with $1<\psi<\pi$. Let $\mathcal{C}$ be the subset of chains in the poset interval $[1, \pi]$ where the second-highest element is $\psi$. Then

$$
\sum_{c \in \mathcal{C}}(-1)^{|c|}=-\mu[\psi]
$$

Proof. If we remove $\pi$ from the chains in $\mathcal{C}$, then we have all of the chains in the poset interval $[1, \psi]$, and the Hall sum of these chains is, by definition, $\mu[\psi]$. It follows that the Hall sum of the chains in $\mathcal{C}$ is $-\mu[\psi]$.

Corollary 7. Given a permutation $\pi$, and a set of permutations $S$ where every $\sigma \in S$ satisfies $1<\sigma<\pi$, then if $\mathcal{C}$ is the set of chains in the poset interval $[1, \pi]$ where the second-highest element is in $S$, then the Hall sum of $\mathcal{C}$ is $-\sum_{\sigma \in S} \mu[\sigma]$.

Proof. First, partition $\mathcal{C}$ based on the second-highest element, and then apply Lemma 6 to each partition. 
When discussing chains, in general we will only be interested in a small subset of the chain containing two or three elements. We say that a segment of some chain $c$ is a non-empty subset of the elements in $c$ with the property that any element not in the segment is either less than every element in the segment, or is greater than every element in the segment.

In our proofs, given a set of chains $\mathcal{C}$, and a chain $c \in \mathcal{C}$, we will frequently want to construct a chain $c^{\prime}$ by using a parity-reversing involution $\Phi$. Strictly speaking, $\Phi$ is a function that maps a set of permutations (which is a chain) to a set of permutations (which may not be a chain). As examples, if $\Phi(c)$ removes the largest or smallest element of $c$, or adds an element so that $\Phi(c)$ does not have a total order, then $\Phi(c)$ is not a chain. To show that $\Phi$ is a parity-reversing involution we will need to show that $\Phi(c)$ is a chain in $\mathcal{C}$, and that $c$ and $\Phi(c)$ have opposite parities. In our discussions, we will typically set $c^{\prime}=\Phi(c)$, and then show that the set of permutations $c^{\prime}$ is a chain. We will then, without further comment, treat $c^{\prime}$ as a chain.

\section{2413-Balloons}

In this section we define the vocabulary and notation specific to this paper. We also present some general results which will be used in later sections.

Given a non-empty permutation $\beta$, the 2413-balloon of $\beta$ is the permutation formed by inserting $\beta$ into the centre of 2413 , which we write as $2413 \odot \beta$. Formally, we have

$$
(2413 \odot \beta)_{i}= \begin{cases}2 & \text { if } i=1 \\ |\beta|+4 & \text { if } i=2 \\ \beta_{i-2}+2 & \text { if } i>2 \text { and } i \leqslant|\beta|+2 \\ 1 & \text { if } i=|\beta|+3 \\ |\beta|+3 & \text { if } i=|\beta|+4\end{cases}
$$

Figure 1(a) shows $2413 \odot \beta$.

The balloon operation as defined has to be right-associative and the definition given does not support overriding right-associativity. In other words, $2413 \odot 2413 \odot \beta$ must be $2413 \odot(2413 \odot \beta)$, and $(2413 \odot 2413) \odot \beta$ is not defined. In Section 7 we suggest how the balloon operation could be generalized.

Given some $\pi=2413 \odot \beta$, if $\beta$ is itself a 2413-balloon, so $\pi=2413 \odot 2413 \odot \gamma$, then we say that $\pi$ is a double 2413-balloon. Figure 1(b) shows a double 2413-balloon.

Remark 8. We note that we can write $2413 \odot \beta$ as the inflation $25314[1,1, \beta, 1,1]$ (see Albert and Atkinson [1] for further details of inflations). In this paper we use balloon notation, as we feel that this leads to a simpler exposition.

If we have $\pi=2413 \odot \beta$, and we have some $\sigma$ with $\beta \leqslant \sigma<\pi$, we will frequently want to represent $\sigma$ in terms of sub-permutations of 2413 and the permutation $\beta$. We start by 


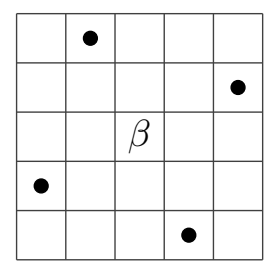

(a)

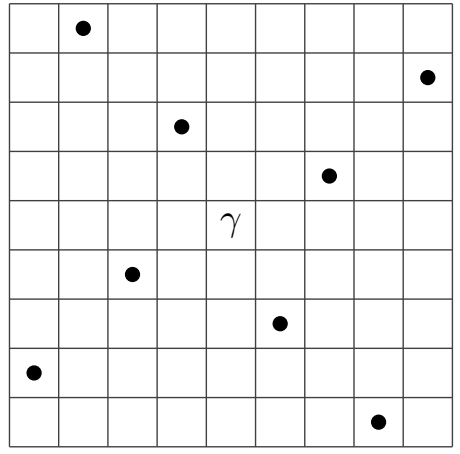

(b)

Figure 1: (a) The 2413-balloon $2413 \odot \beta$ and (b) the double 2413-balloon $2413 \odot 2413 \odot \gamma$.

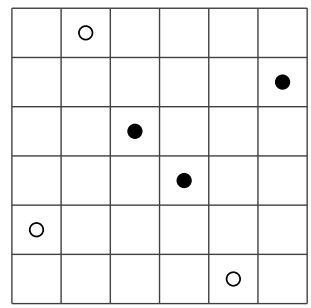

Figure 2: An embedding of $213=\overline{241} 3 \odot 21$ in $264315=2413 \odot 21$.

colouring the extremal points of $\pi$ red, and all remaining points black. Note that the red points are a 2413 permutation, and the black points are $\beta$.

Now consider a specific embedding of $\sigma$ into $\pi$, where we use all of the black points $(\beta)$. If the embedding is monochromatic $(\sigma=\beta)$ then we require no special notation. If the embedding is not monochromatic, then it must be the case that only some of the red points are used. We take 2413, and mark the red points that are unused with an overline, and then write $\sigma$ using our balloon notation. As an example of this, if $\pi=2413 \odot 21=264315$, and $\sigma=213$, then we could represent $\sigma$ as $\overline{241} 3 \odot 21$. This example is shown in Figure 2 . We can see that if $\beta \leqslant \sigma<2413 \odot \beta$, and $\beta$ is not monotonic (i.e., not the identity permutation or its reverse), then there is a unique way to represent $\sigma$ using this notation.

If we have $\pi=2413 \odot \beta$, and $\sigma$ is a permutation such that $\beta \leqslant \sigma<\pi$, then we say that $\sigma$ is a reduction of $\pi$. If $\sigma$ is a reduction of $\pi=2413 \odot \beta$, and there is no $\eta$ with $|\eta|<|\beta|$ such that $\sigma$ is a reduction of $2413 \odot \eta$, then we say that $\sigma$ is a proper reduction of $\pi$. A reduction of $\pi$ that is not a proper reduction is an improper reduction.

The following case-by-case analysis shows the improper reductions (of $\pi$ ) based on the form of $\beta$. 


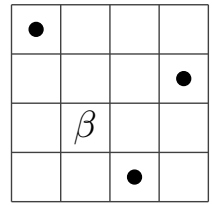

$\overline{2} 413 \odot \beta$

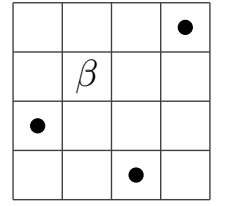

$2 \overline{4} 13 \odot \beta$

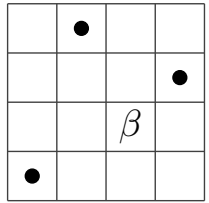

$24 \overline{1} 3 \odot \beta$

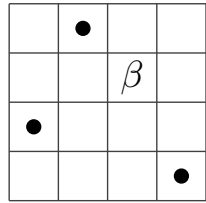

$241 \overline{3} \odot \beta$

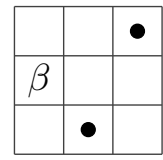

$\overline{24} 13 \odot \beta$

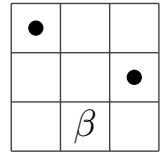

$\overline{2} 4 \overline{1} 3 \odot \beta$

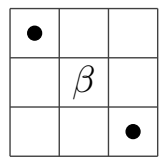

$\overline{2} 41 \overline{3} \odot \beta$

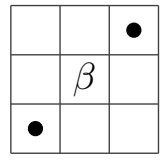

$2 \overline{413} \odot \beta$

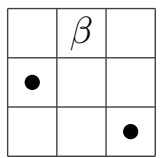

$2 \overline{4} 1 \overline{3} \odot \beta$

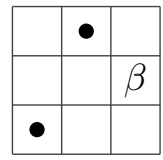

$24 \overline{13} \odot \beta$

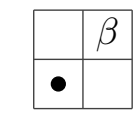

$2 \overline{413} \odot \beta$

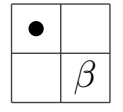

$\overline{2} 4 \overline{13} \odot \beta$

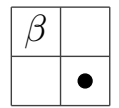

$\overline{24} 1 \overline{3} \odot \beta$

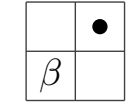

$\overline{241} 3 \odot \beta$

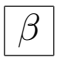

$\beta$

Figure 3: Reductions of $\pi=2413 \odot \beta$. Some may not be proper reductions, depending on $\beta$.

- If $\beta$ is a 2413 -balloon, then $\beta$ is the only improper reduction of $\pi$.

- If $\beta$ is not a 2413-balloon, and $\beta$ has no corners, then there are no improper reductions of $\pi$.

- If $\beta$ has one corner, then there are four improper reductions of $\pi$. As an example, if $\beta=1 \oplus \gamma$, then the improper reductions of $\pi$ are $\overline{24} 13 \odot \beta, \overline{24} 1 \overline{3} \odot \beta, \overline{2413} \odot \beta$, and $\beta$.

- If $\beta$ has two corners, then there are seven improper reductions of $\pi$. As an example, if $\beta=1 \oplus \gamma \oplus 1$, then the improper reductions are $\overline{24} 13 \odot \beta, 24 \overline{13} \odot \beta, 2 \overline{413} \odot \beta$, $\overline{2} 4 \overline{13} \odot \beta, \overline{24} 1 \overline{3} \odot \beta, \overline{241} 3 \odot \beta$, and $\beta$.

The set of permutations that are proper reductions of $\pi$ is written as $\mathrm{R}_{\pi}$. Figure 3 shows all the reductions (proper and improper) of $\pi=2413 \odot \beta$.

The strategy that we will use in Sections 4 and 6 is to partition the chains in the poset interval $[1, \pi]$ into three sets, $\mathcal{R}, \mathcal{G}$, and $\mathcal{B}$. We then show that there are parity-reversing involutions on the sets $\mathcal{G}$ and $\mathcal{B}$, and therefore, by Corollary 5 , the Hall sum for each of these sets is zero, and so $\mu[\pi]$ is given by the Hall sum of the set $\mathcal{R}$. Finally, we show that the Hall sum of $\mathcal{R}$ can be written in terms of $\mu[\beta]$. 


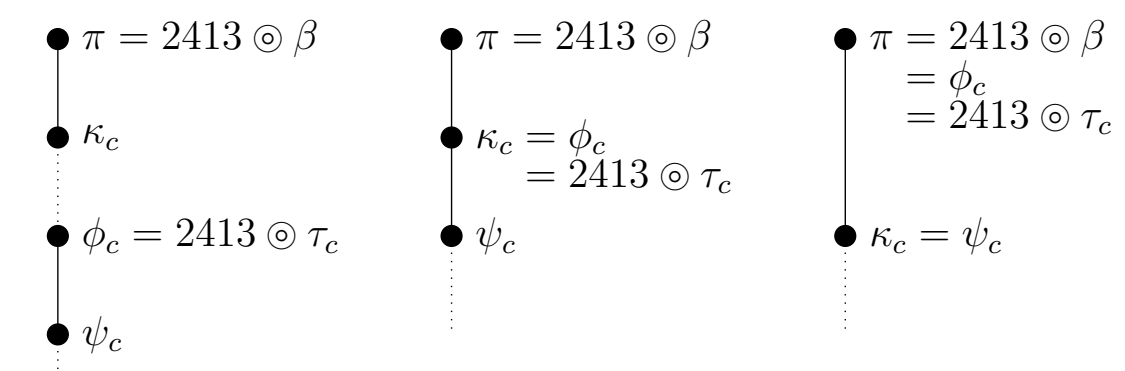

Figure 4: Examples of chains, showing some possible relationships between $\pi, \kappa_{c}, \phi_{c}$, and $\psi_{c}$.

The chains in $\mathcal{R}$ are those chains where the second-highest element is a proper reduction of $\pi$, so if $\kappa_{c}$ is the second-highest element of a chain $c$, then $c \in \mathcal{R}$ if and only if $\kappa_{c} \in \mathrm{R}_{\pi}$. Note that, as mentioned earlier, the members of $\mathrm{R}_{\pi}$, and hence the chains in $\mathcal{R}$, depend on the form of $\pi$. It is easy to see that for any permutation $\sigma \in \mathrm{R}_{\pi}$ we must have $|\sigma| \geqslant|\beta|$.

We have some results that are independent of $R_{\pi}$, and, once we have given some some further definitions, we present these in the current section to avoid repetition.

Let $\pi$ be a 2413-balloon, and let $c$ be any chain in the poset interval $[1, \pi]$.

Since the top of the chain is, by definition, a 2413-balloon, it follows that $c$ has a unique maximal segment that includes the element $\pi$, where every element in the segment is a 2413-balloon. We call the smallest element in this segment the least 2413-balloon ${ }^{1}$.

Further, since the permutation 1 is not a 2413-balloon, it follows that $c$ has an element that is immediately below the least 2413-balloon in the chain, and we call this element the pivot.

We define $\phi_{c}$ to be the least 2413-balloon in $c, \psi_{c}$ to be the pivot in $c, \tau_{c}$ to be the permutation that satisfies $2413 \odot \tau_{c}=\phi_{c}$, and $\kappa_{c}$ to be the second-highest element of $c$. Note that $\phi_{c}$ and $\psi_{c}$ must be distinct, but we can have $\tau_{c}=\psi_{c}$. Further, $\kappa_{c}$ is independent, and may be the same as $\phi_{c}, \psi_{c}$ or $\tau_{c}$. Figure 4 shows some example chains, highlighting these elements.

We are now in a position to give a definition of the sets $\mathcal{R}, \mathcal{G}$, and $\mathcal{B}$. This definition depends on the set of proper reductions of $\pi, \mathrm{R}_{\pi}$, which, as stated earlier, depends on the form of $\beta$.

\footnotetext{
${ }^{1}$ The name should really be "least 2413-balloon in the chain that has only 2413 -balloons above it".
} 
Let $\mathcal{C}$ be the set of chains in the poset interval $[1, \pi]$. We define subsets of $\mathcal{C}$ as follows:

$$
\begin{aligned}
& \mathcal{R}=\left\{c: c \in \mathcal{C} \text { and } \kappa_{c} \in \mathrm{R}_{\pi}\right\}, \\
& \mathcal{G}=\left\{c: c \in \mathcal{C} \backslash \mathcal{R} \text { and } \psi_{c} \leqslant 2413\right\} \\
& \mathcal{B}=\{c: c \in \mathcal{C} \backslash(\mathcal{R} \cup \mathcal{G})\} .
\end{aligned}
$$

Clearly, every chain in $[1, \pi]$ is included in exactly one of these subsets, and so these sets are a partition of the chains.

Given a pivot $\psi_{c}$, there is a unique permutation $\eta_{c}$ which we call the core of $\psi_{c}$. In essence, $\eta_{c}$ is the smallest permutation such that $\psi_{c}<2413 \odot \eta_{c}$. To determine the core, we use the following algorithm:

$$
\begin{aligned}
& \text { If } \psi_{c} \text { can be written as } 1 \ominus((\eta \ominus 1) \oplus 1) \text { or } \quad((1 \oplus \eta) \ominus 1) \oplus 1 \\
& \text { or } 1 \oplus(1 \ominus(\eta \oplus 1)) \text { or }(1 \oplus(1 \ominus \eta)) \ominus 1 \text {, } \\
& \text { then set } \eta_{c}=\eta \text {. }
\end{aligned}
$$

Otherwise, if $\psi_{c}$ can be written as $(\eta \ominus 1) \oplus 1$ or $1 \ominus(\eta \oplus 1)$ or $1 \ominus \eta \ominus 1$

$$
\text { or } 1 \oplus \eta \oplus 1 \text { or }(1 \oplus \eta) \ominus 1 \text { or } 1 \oplus(1 \ominus \eta) \text {, }
$$

then set $\eta_{c}=\eta$.

Otherwise, if $\psi_{c}$ can be written as $1 \oplus \eta$ or $1 \ominus \eta$ or $\eta \ominus 1$ or $\eta \oplus 1$,

then set $\eta_{c}=\eta$.

Otherwise, set $\eta_{c}=\psi_{c}$.

Since we have $\psi_{c}<\phi_{c}=2413 \odot \tau_{c}$, it is easy to see that $\eta_{c} \leqslant \tau_{c}$. Note that $2413 \odot \eta_{c}$ is the smallest 2413-balloon that contains $\psi_{c}$.

We now define two functions, one for each of $\mathcal{G}$ and $\mathcal{B}$, which will give us parity-reversing involutions.

$$
\begin{aligned}
& \Phi_{\mathcal{G}}(c)= \begin{cases}c \backslash\{2413\} & \text { If } \psi_{c}=2413 \\
c \cup\{2413\} & \text { If } \psi_{c}<2413\end{cases} \\
& \Phi_{\mathcal{B}}(c)= \begin{cases}c \backslash\left\{2413 \odot \eta_{c}\right\} & \text { If } \eta_{c}=\tau_{c} \\
c \cup\left\{2413 \odot \eta_{c}\right\} & \text { If } \eta_{c}<\tau_{c}\end{cases}
\end{aligned}
$$

Remark 9. If we were to allow the ballooning of the empty permutation $\epsilon$, and then treat 2413 as $2413 \odot \epsilon$ then $\Phi_{\mathcal{G}}(c)$ is subsumed by $\Phi_{\mathcal{B}}(c)$. Doing this, however, introduces additional complications in later proofs, and so we prefer two involutions. 
For $\Phi_{\mathcal{G}}(c)$ to be a parity-reversing involution on $\mathcal{G}$, we need to show that if $c \in \mathcal{G}$, then $\Phi_{\mathcal{G}}(c)$ is a chain, that $\Phi(c) \in \mathcal{G}$, and that $c$ and $\Phi(c)$ have different parities. It is easy to see that this last condition is true. A similar comment applies to $\Phi_{\mathcal{B}}(c)$ and $\mathcal{B}$.

For $\Phi_{\mathcal{G}}(c)$ we can show that all the conditions hold for any $\mathrm{R}_{\pi}$, regardless of the form of $\beta$. For $\Phi_{\mathcal{B}}(c)$ we show that some weaker conditions hold for an arbitrary subset of the reductions of $\pi$, and then, when we have an explicit set of proper reductions, we show that all conditions hold. The following Lemma gives us a result that applies to $\Phi_{\mathcal{G}}(c)$ and $\Phi_{\mathcal{B}}(c)$ for any $\mathrm{R}_{\pi}$, and we will use this result in both Section 4 and Section 6.

Lemma 10. Let $\pi=2413 \odot \beta$, with $|\beta|>4$, and let $\mathcal{R}, \mathcal{G}$, and $\mathcal{B}$ be as defined above.

(a) If $c \in \mathcal{G}$, then $\Phi_{\mathcal{G}}(c) \in \mathcal{G}$.

(b) If $c \in \mathcal{B}$, with $\eta_{c}=\tau_{c}$, and $\Phi_{\mathcal{B}}(c)$ is a chain, then $\Phi_{\mathcal{B}}(c) \in \mathcal{B} \cup \mathcal{R}$.

(c) If $c \in \mathcal{B}$, with $\eta_{c}<\tau_{c}$, then $\Phi_{\mathcal{B}}(c) \in \mathcal{B} \cup \mathcal{R}$.

Proof. Case (a). First, assume that $c \in \mathcal{G}$ with $\psi_{c}=2413$. Then $c$ contains a segment $2413<2413 \odot \tau_{c}$, and $c^{\prime}=\Phi_{\mathcal{G}}(c)=c \backslash\{2413\}$. We can see that $c^{\prime}$ is a chain, as 2413 is neither the smallest nor the largest entry in $c^{\prime}$. Further, $\psi_{c^{\prime}}<2413$. Since $|\beta|>4$, and $\left|\psi_{c^{\prime}}\right|<4$ we must have $c^{\prime} \notin \mathcal{R}$, and therefore $c^{\prime} \in \mathcal{G}$.

Now assume that $c \in \mathcal{G}$ with $\psi_{c}<2413$. Then $c$ contains a segment $\psi_{c}<2413 \odot \tau_{c}$, and $c^{\prime}=\Phi_{\mathcal{G}}(c)=c \cup\{2413\}$. We can see that $c^{\prime}$ is a chain, since $\psi_{c}<2413<2413 \odot \tau_{c}$, and further, $\psi_{c^{\prime}}=2413$. Since $|\beta|>4$, and $\left|\psi_{c^{\prime}}\right|=4$ we must have $c^{\prime} \notin \mathcal{R}$, and therefore $c^{\prime} \in \mathcal{G}$.

Case (b). Let $c$ be a chain in $\mathcal{B}$, with $\eta_{c}=\tau_{c}$. Then $c$ contains a segment $\psi_{c}<2413 \odot \tau_{c}$, and $c^{\prime}=\Phi_{\mathcal{B}}(c)=c \backslash\left\{2413 \odot \tau_{c}\right\}$. If $\tau_{c}=\beta$, then $c^{\prime}$ is not a chain, so we must have $\tau_{c}<\beta$, and therefore $c^{\prime}$ is a chain that contains a segment $\psi_{c}<2413 \odot \gamma$, with $\tau_{c}<\gamma$. Now, $\psi_{c}$ is the pivot of $c^{\prime}$, so we cannot have $c^{\prime} \in \mathcal{G}$ as this would imply that $c \in \mathcal{G}$, which is a contradiction. Thus either $c^{\prime} \in \mathcal{R}$ or $c^{\prime} \in \mathcal{B}$.

Case (c). Let $c$ be a chain in $\mathcal{B}$, with $\eta_{c}<\tau_{c}$. Then $c$ contains a segment $\psi_{c}<2413 \odot \tau_{c}$, and $c^{\prime}=\Phi_{\mathcal{B}}(c)=c \cup\left\{2413 \odot \eta_{c}\right\}$. We can see that $c^{\prime}$ is a chain since $\psi_{c}<2413 \odot \eta_{c}<$ $2413 \odot \tau_{c}$. Now, $\psi_{c}$ is the pivot of $c^{\prime}$, so we cannot have $c^{\prime} \in \mathcal{G}$ as this would imply that $c \in \mathcal{G}$, which is a contradiction. So either $c^{\prime} \in \mathcal{R}$ or $c^{\prime} \in \mathcal{B}$.

We now have

Observation 11. If $\pi=2413 \odot \beta$, with $|\beta|>4$, then to show that $\Phi_{\mathcal{B}}$ is a parity-reversing involution on $\mathcal{B}$ it is sufficient to show that:

(a) If $c \in \mathcal{B}$ and $\eta_{c}=\tau_{c}$, then $\Phi_{\mathcal{B}}(c)$ is a chain, and $\Phi_{\mathcal{B}}(c) \notin \mathcal{R}$. 
(b) If $c \in \mathcal{B}$, and $\eta_{c}<\tau_{c}$, then $\Phi_{\mathcal{B}}(c) \notin \mathcal{R}$.

Further, if $\Phi_{\mathcal{B}}$ is a parity-reversing involution on $\mathcal{B}$, then $\mu[\pi]=-\sum_{\sigma \in \mathrm{R}_{\pi}} \mu[\sigma]$.

Proof. Combining (a) and (b) above with cases (b) and (c) of Lemma 10 gives us that $\Phi_{\mathcal{B}}$ is a parity-reversing involution on $\mathcal{B}$.

This now gives us that $\sum_{c \in \mathcal{B}}(-1)^{|c|}=0$. From Lemma 10, we have $\sum_{c \in \mathcal{G}}(-1)^{|c|}=0$, so we must have $\mu[\pi]=\sum_{c \in \mathcal{R}}(-1)^{|c|}$. Since the chains in $\mathcal{R}$ are defined by the secondhighest element $\left(\kappa_{c}\right)$ being in $\mathrm{R}_{\pi}$, the final part of the observation follows by applying Corollary 7.

\section{The Möbius function of double 2413-balloons}

We are now able to state and prove our first major result.

Theorem 12. Let $\pi=2413 \odot \beta$, where $\beta$ is a 2413-balloon, Then $\mu[\pi]=2 \mu[\beta]$.

Proof. Note that $\beta \notin \mathrm{R}_{\pi}$, and further that $|\beta|>4$, since $\beta$ is a 2413-balloon.

Using Observation 11 , we will show that $\Phi_{\mathcal{B}}$ is a parity-reversing involution on $\mathcal{B}$. Once we have shown that we have parity-reversing involutions, we will then show how to express the Hall sum of $\mathcal{R}$ in terms of $\mu[\beta]$.

Proof that $\Phi_{\mathcal{B}}$ is a parity-reversing involution on $\mathcal{B}$. Let $c$ be a chain in $\mathcal{B}$.

First, assume that $\eta_{c}=\tau_{c}$. If $\tau_{c}=\beta$, then either $\psi_{c}$ is a proper reduction of $\pi$, or $\psi_{c}=\beta$. In the first case, $c \in \mathcal{R}$, and in the second case $\psi_{c}$ is a 2413-balloon, and these are both contradictions. Thus we must have $\tau_{c}<\beta$, and so there is at least one permutation in $c$ greater than $\phi_{c}$. It follows that $c^{\prime}$ is a chain. We now show that $c^{\prime} \notin \mathcal{R}$. Assume, to the contrary, that $c^{\prime} \in \mathcal{R}$ which implies that $\psi_{c}$ is a proper reduction of $\pi$. But now we have $\eta_{c}=\beta$, which is a contradiction, so $\psi_{c}$ is not a proper reduction of $\pi$, therefore $c^{\prime} \notin \mathcal{R}$.

Now assume that $\eta_{c}<\tau_{c}$. Let $c^{\prime}=\Phi_{\mathcal{B}}(c)=c \cup\left\{2413 \odot \eta_{c}\right\}$. We know by Lemma 10 that this is a chain. Either $\kappa_{c}=\kappa_{c^{\prime}}$, or $\kappa_{c^{\prime}}$ is a 2413-balloon. If $\kappa_{c}=\kappa_{c^{\prime}}$, then $c^{\prime} \notin \mathcal{R}$. If $\kappa_{c^{\prime}}$ is a 2413-balloon, then $\kappa_{c^{\prime}} \notin \mathrm{R}_{\pi}$, so $c^{\prime} \notin \mathcal{R}$. Thus we must have $c^{\prime} \notin \mathcal{R}$.

So now we have that if $c \in \mathcal{B}$ and $\eta_{c}=\tau_{c}$, then $\Phi_{\mathcal{B}}(c)$ is a chain; and that for any $c \in \mathcal{B}$, $\Phi_{\mathcal{B}}(c) \in \mathcal{B}$. It follows that $\Phi_{\mathcal{B}}$ is a parity-reversing involution on $\mathcal{B}$.

We now have that $\Phi_{\mathcal{G}}$ and $\Phi_{\mathcal{B}}$ are parity-reversing involutions on $\mathcal{G}$ and $\mathcal{B}$ respectively. It follows from Observation 11 that $\mu[\pi]=-\sum_{\sigma \in \mathrm{R}_{\pi}} \mu[\sigma]$. We now show how to express $\mu[\sigma]$, where $\sigma \in \mathrm{R}_{\pi}$, in terms of $\mu[\beta]$. 
We start by noting that since $\beta$ is a 2413 -balloon, then $\beta$ has no corners. Now, take the case where $\sigma=\overline{2} 413 \odot \beta$, which is the first permutation in Figure 3. Note that we can write $\sigma=1 \ominus((\beta \ominus 1) \oplus 1)$. Applying Lemma 2 to the outermost three points in $\sigma$ (those from the 2413$)$, we find that $\mu[\sigma]=-\mu[\beta]$. The other cases are similar, and this gives us: $^{2}$

\begin{tabular}{|c|c|c|c|c|}
\hline$\sigma$ & $\mu[\sigma]$ & $\mu[\sigma]$ & $\sigma$ & $\mu[\sigma]$ \\
\hline$\overline{2} 413 \odot \beta$ & $-\mu[\beta]$ & $\overline{24} 13 \odot \beta \quad \mu[\beta]$ & $2 \overline{413} \odot \beta$ & $-\mu[\beta]$ \\
\hline $2 \overline{4} 13 \odot \beta$ & $-\mu[\beta]$ & $\overline{2} 4 \overline{1} 3 \odot \beta \quad \mu[\beta]$ & $\overline{2} 4 \overline{13} \odot \beta$ & $-\mu[\beta]$ \\
\hline $24 \overline{1} 3$ ○ $\beta$ & $-\mu[\beta]$ & $\overline{2} 41 \overline{3} \odot \beta \quad \mu[\beta]$ & $\overline{24} 1 \overline{3} \odot \beta$ & $-\mu[\beta]$ \\
\hline $241 \overline{3} \odot \beta$ & $-\mu[\beta]$ & $2 \overline{41} 3 \odot \beta \quad \mu[\beta]$ & $\overline{241} 3 \odot \beta$ & $-\mu[\beta]$ \\
\hline & & $2 \overline{4} 1 \overline{3} \odot \beta \quad \mu[\beta]$ & & \\
\hline & & $24 \overline{13} \odot \beta \quad \mu[\beta]$ & & \\
\hline
\end{tabular}

It is now easy to see that

$$
\sum_{\sigma \in \mathrm{R}_{\pi}} \mu[\sigma]=-2 \mu[\beta]
$$

and the result follows directly.

\section{The growth of the Möbius function}

We define $\max _{\mu}(n)=\max \{|\mu[\pi]|:|\pi|=n\}$. Previous work in [5] and [6] has shown that the growth of $\max _{\mu}(n)$ is at least polynomial. We will show that the growth is at least exponential. We have

Theorem 13. For all $n, \max _{\mu}(n) \geqslant 2^{\lfloor n / 4\rfloor-1}$.

Proof. We start by a defining a function to construct a permutation of length $n$.

$$
\pi^{(n)}= \begin{cases}1 & \text { If } n=1 \\ 12 & \text { If } n=2 \\ 132 & \text { If } n=3 \\ 2413 & \text { If } n=4 \\ 2413 \odot \pi^{(n-4)} & \text { Otherwise }\end{cases}
$$

Note that for $n>8, \pi^{(n)}$ is a double 2413-balloon. It is simple to calculate $\mu\left[\pi^{(n)}\right]$ for $n=1, \ldots, 8$, and these values are given below.

\footnotetext{
${ }^{2}$ This table is slightly redundant, as the entries are determined by the parity of the "red" points. We include it as later results have similar tables where some values of $\mu[\sigma]$ are zero, and this gives a consistent presentation.
} 


$$
\begin{aligned}
\mu\left[\pi^{(1)}\right] & =\mu[1]=1, & \mu\left[\pi^{(5)}\right] & =\mu[25314]=4, \\
\mu\left[\pi^{(2)}\right] & =\mu[12]=-1, & \mu\left[\pi^{(6)}\right] & =\mu[263415]=-1, \\
\mu\left[\pi^{(3)}\right] & =\mu[132]=1, & \mu\left[\pi^{(7)}\right] & =\mu[2735416]=1, \\
\mu\left[\pi^{(4)}\right] & =\mu[2413]=-3, & \mu\left[\pi^{(8)}\right] & =\mu[28463517]=-6 .
\end{aligned}
$$

These values match Theorem 13, and so this is true for $n \leqslant 8$. For $n>8, \mu\left[\pi^{(n)}\right]=$ $2 \mu\left[\pi^{(n-4)}\right]$ by Theorem 12 , and the result follows immediately.

Remark 14 . It is easy to see that, with the definitions above, the only simple permutations that can be contained in $\pi^{(n)}$ are 1, 12, 21, 2413, and 25314. This answers Problem 4.4 in [5], which asks whether $\mu[\pi]$ is bounded on a hereditary class which contains only finitely many simple permutations, as, by Theorem 13, we have unbounded growth, but only finitely many simple permutations.

If we repeat the ballooning process, as we do in $\pi^{(n)}$, then the permutation plot is rather striking. We illustrate this in Figure 5, which shows $\pi^{(21)}$.

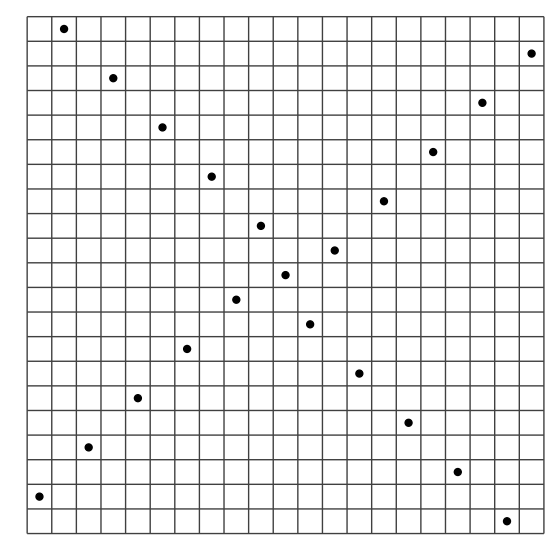

Figure 5: A permutation plot showing $\pi^{(21)}$.

\section{The Möbius function of 2413-balloons}

Theorem 12 gives us an expression for the value of the Möbius function $\mu[\pi]$ when $\pi$ is a double 2413-balloon. We expand on this to find an expression for the Möbius function $\mu[\pi]$ when $\pi$ is any 2413 -balloon.

We start with a Lemma that handles the case where $\beta$ is not a 2413-balloon, and has more than four points. The structure of our proof is similar to that of Theorem 12, but we present a complete argument to aid readability.

We will show 
Lemma 15. Let $\pi=2413 \odot \beta$, where $\beta$ is not a 2413-balloon, and $|\beta|>4$. Then $\mu[\pi]=\mu[\beta]$.

Proof. First note that if $\beta$ is monotonic, then by Corollary 4 we have $\mu[\beta]=0=\mu[\pi]$. For the remainder of this proof, we assume that $\beta$ is not monotonic.

If $\beta$ has one corner, then without loss of generality, we can assume by symmetry that $\beta=1 \oplus \gamma$. Similarly, if $\beta$ has two corners, then we can assume that $\beta=1 \oplus \gamma \oplus 1$.

As before, we will use Observation 11. We will show that $\Phi_{\mathcal{B}}$ is a parity-reversing involution on $\mathcal{B}$. Once we have shown that we have parity-reversing involutions, we will then show how to express the Hall sum of $\mathcal{R}$ in terms of $\mu[\beta]$.

The proper reductions of $\pi$ depend on the number of corners of $\beta$. Below we list the improper reductions of $\pi$ for each case.

\begin{tabular}{lr}
\hline Corners in $\beta$ & Improper reductions of $\pi$ \\
\hline $\begin{array}{l}\text { No corners } \\
\text { One corner }(\beta=1 \oplus \gamma)\end{array}$ & $\begin{array}{c}\text { None. } \\
\text { Two corners }(\beta=1 \oplus \gamma \oplus 1)\end{array}$ \\
& $\overline{24} 13 \odot \beta, \overline{24} 1 \overline{3} \odot \beta, \overline{2413} \odot \beta$, and $\beta$. \\
$\overline{2413} \odot \beta, \overline{24} 4 \overline{13} \odot \beta, \overline{24} 1 \overline{3} \odot \beta, \overline{2413} \odot \beta$, \\
and $\beta$.
\end{tabular}

Proof that $\Phi_{\mathcal{B}}$ is a parity-reversing involution on $\mathcal{B}$. Let $c$ be a chain in $\mathcal{B}$.

First, assume that $\eta_{c}=\tau_{c}$, so $c^{\prime}=c \backslash\left\{2413 \odot \tau_{c}\right\}$. We start by showing that $c^{\prime}$ is a valid chain. Assume otherwise, which implies $\tau_{c}=\beta$.

If $\beta$ has no corners, then $\psi_{c} \in \mathrm{R}_{\pi}$, so $c \in \mathcal{R}$, which is a contradiction.

If $\beta$ has one corner, then either $\psi_{c} \in \mathrm{R}_{\pi}$, which is a contradiction, or $\psi_{c} \notin \mathrm{R}_{\pi}$. In the latter case, assume, without loss of generality, that $\beta=1 \oplus \gamma$. Then $\overline{24} 13 \odot \beta=2 \overline{4} 13 \odot \gamma$, $\overline{24} 1 \overline{3} \odot \beta=2 \overline{4} 1 \overline{3} \odot \gamma, \overline{2413} \odot \beta=2 \overline{41} 3 \odot \gamma$, and $\beta=2 \overline{413} \odot \gamma$. Thus in all cases where $\psi_{c} \notin \mathrm{R}_{\pi}$, we have that $\eta_{c}$ is not minimal, which is a contradiction.

Finally, if $\beta$ has two corners, then either $\psi_{c} \in \mathrm{R}_{\pi}$, which is a contradiction, or $\psi_{c} \notin \mathrm{R}_{\pi}$. The latter case implies that $\psi_{c}=\beta$, and then we have that either $\psi_{c}=1 \oplus \gamma \oplus 1=2 \overline{41} 3 \odot \gamma$ or $\psi_{c}=1 \ominus \gamma \ominus 1=\overline{2} 41 \overline{3} \odot \gamma$, so $\eta_{c}$ is not minimal, which is a contradiction.

Thus we have that $c^{\prime}$ must be a chain, and, moreover, $\tau_{c} \neq \beta$.

We now show that $c^{\prime} \notin \mathcal{R}$. Assume, to the contrary, that $c^{\prime} \in \mathcal{R}$ which implies that $\psi_{c}$ is a proper reduction of $\pi$. But now we have $\eta_{c}=\beta$, but this would give $\tau_{c}=\beta$, which is a contradiction, therefore $c^{\prime} \notin \mathcal{R}$.

Now assume that $\eta_{c}<\tau_{c}$. Let $c^{\prime}=\Phi_{\mathcal{B}}(c)=c \cup\left\{2413 \odot \eta_{c}\right\}$, and we know from Lemma 10 that $c^{\prime}$ is a chain. Now either $\kappa_{c}=\kappa_{c^{\prime}}$, or $\kappa_{c^{\prime}}=2413 \odot \eta_{c}$ is a 2413-balloon. In either case 
we have $c^{\prime} \notin \mathcal{R}$.

So if $c \in \mathcal{B}$, then $\Phi_{\mathcal{B}}(c)$ is a chain in $\mathcal{B}$, and thus $\Phi_{\mathcal{B}}$ is a parity-reversing involution.

We have shown that $\Phi_{\mathcal{G}}$ and $\Phi_{\mathcal{B}}$ are parity-reversing involutions on $\mathcal{G}$ and $\mathcal{B}$ respectively. It follows from Observation 11 that $\mu[\pi]=-\sum_{\sigma \in \mathrm{R}_{\pi}} \mu[\sigma]$. We now show how to express $\mu[\sigma]$, where $\sigma \in \mathrm{R}_{\pi}$ in terms of $\mu[\beta]$. We use a similar mechanism to that used in Theorem 12 . There are some additional considerations where $\beta$ has one or two corners.

As an example, take the case where $\sigma=2 \overline{4} 13 \odot \beta$, and $\beta$ has one corner, and so, by our assumption, can be written as $1 \oplus \gamma$. We can write $\sigma=((1 \oplus \beta) \ominus 1) \oplus 1$, and expanding $\beta$ we have $\sigma=((1 \oplus 1 \oplus \gamma) \ominus 1) \oplus 1$, Applying Lemma 2 to the outermost two points in $\sigma$, we find that $\mu[\sigma]=\mu[1 \oplus 1 \oplus \gamma]$, and by Lemma 1 we now have $\mu[\sigma]=0$. Because of this, our analysis depends on the number of corners of $\beta$, and we consider each case separately below.

If $\beta$ has no corners, then we have

$$
\begin{array}{lr}
\sigma & \mu[\sigma] \\
\hline \overline{2} 413 \odot \beta & -\mu[\beta] \\
2 \overline{4} 13 \odot \beta & -\mu[\beta] \\
24 \overline{1} 3 \odot \beta & -\mu[\beta] \\
241 \overline{3} \odot \beta & -\mu[\beta]
\end{array}
$$

\begin{tabular}{ll}
$\sigma$ & $\mu[\sigma]$ \\
\hline$\overline{24} 13 \odot \beta$ & $\mu[\beta]$ \\
$\overline{2} 4 \overline{1} 3 \odot \beta$ & $\mu[\beta]$ \\
$\overline{2} 41 \overline{3} \odot \beta$ & $\mu[\beta]$ \\
$2 \overline{41} 3 \odot \beta$ & $\mu[\beta]$ \\
$2 \overline{4} 1 \overline{3} \odot \beta$ & $\mu[\beta]$ \\
$24 \overline{13} \odot \beta$ & $\mu[\beta]$
\end{tabular}

\begin{tabular}{lr}
$\sigma$ & $\mu[\sigma]$ \\
\hline $2 \overline{413} \odot \beta$ & $-\mu[\beta]$ \\
$\overline{2} 4 \overline{13} \odot \beta$ & $-\mu[\beta]$ \\
$\overline{24} 1 \overline{3} \odot \beta$ & $-\mu[\beta]$ \\
$\overline{2413} \odot \beta$ & $-\mu[\beta]$ \\
$\beta$ & $\mu[\beta]$
\end{tabular}

If $\beta$ has one corner, under our assumption that $\beta=1 \oplus \gamma$, we have

\begin{tabular}{lr}
$\sigma$ & $\mu[\sigma]$ \\
\hline$\overline{2} 413 \odot \beta$ & $-\mu[\beta]$ \\
$2 \overline{4} 13 \odot \beta$ & 0 \\
$24 \overline{1} 3 \odot \beta$ & $-\mu[\beta]$ \\
$241 \overline{3} \odot \beta$ & $-\mu[\beta]$
\end{tabular}

\begin{tabular}{lr}
$\sigma$ & $\mu[\sigma]$ \\
\hline$\overline{2} 4 \overline{1} 3 \odot \beta$ & $\mu[\beta]$ \\
$\overline{2} 41 \overline{3} \odot \beta$ & $\mu[\beta]$ \\
$2 \overline{413} \odot \beta$ & 0 \\
$2 \overline{4} 1 \overline{3} \odot \beta$ & 0 \\
$24 \overline{13} \odot \beta$ & $\mu[\beta]$
\end{tabular}

\begin{tabular}{lr}
$\sigma$ & $\mu[\sigma]$ \\
\hline $2 \overline{413} \odot \beta$ & 0 \\
$\overline{2} 4 \overline{13} \odot \beta$ & $-\mu[\beta]$
\end{tabular}

Finally, if $\beta$ has two corners, under our assumption that $\beta=1 \oplus \gamma \oplus 1$, we have

\begin{tabular}{lrrrr}
$\sigma$ & $\mu[\sigma]$ & & $\sigma$ & $\mu[\sigma]$ \\
\cline { 5 - 6 }$\overline{2} 413 \odot \beta$ & $-\mu[\beta]$ & & $\overline{2} 4 \overline{1} 3 \odot \beta$ & 0 \\
$2 \overline{4} 13 \odot \beta$ & 0 & & $\overline{2} 41 \overline{3} \odot \beta$ & $\mu[\beta]$ \\
$24 \overline{1} 3 \odot \beta$ & 0 & & $2 \overline{41} 3 \odot \beta$ & 0 \\
$241 \overline{3} \odot \beta$ & $-\mu[\beta]$ & & $2 \overline{4} 1 \overline{3} \odot \beta$ & 0
\end{tabular}


In all three cases we have

$$
\sum_{\sigma \in \mathrm{R}_{\pi}} \mu[\sigma]=-\mu[\beta]
$$

and the result follows directly.

We are now in a position to state and prove the main Theorem for this section.

Theorem 16. Let $\pi=2413 \odot \beta$. Then

$$
\mu[\pi]= \begin{cases}4 & \text { If } \beta=1 \\ -6 & \text { If } \beta=2413 \\ 2 \mu[\beta] & \text { If } \beta \text { is a 2413-balloon } \\ \mu[\beta] & \text { Otherwise. }\end{cases}
$$

Proof. The value of $\mu[2413 \odot \beta]$ for the symmetry classes of $\beta$ with $|\beta| \leqslant 4$ are shown below.

\begin{tabular}{lrrrrrr}
$\beta$ & $\mu[\beta]$ & $\mu[2413 \odot \beta]$ & & $\beta$ & $\mu[\beta]$ & $\mu[2413 \odot \beta]$ \\
\cline { 1 - 2 } \cline { 5 - 7 } 1 & 1 & & & 1324 & -1 & -1 \\
12 & -1 & -1 & & 1342 & -1 & -1 \\
123 & 0 & 0 & & 1432 & 0 & 0 \\
132 & 1 & 1 & & 2143 & -1 & -1 \\
1234 & 0 & 0 & & 2413 & -3 & -6 \\
1243 & 0 & 0 & & & &
\end{tabular}

It is easy to see that these values meet Theorem 16. We now combine Theorem 12 and Lemma 15 to complete the proof.

\section{Concluding remarks}

\subsection{Generalising the balloon operator}

Given two permutations $\alpha$ and $\beta$, with lengths $a$ and $b$ respectively, and two integers $i, j$ which satisfy $0 \leqslant i, j \leqslant a$, the $i, j$-balloon of $\beta$ by $\alpha$, written as $\alpha \bigodot_{i, j} \beta$, is the permutation formed by inserting the permutation $\beta$ into $\alpha$ between the $i$-th and $i+1$-th columns of $\alpha$, and between the $j$-th and $j+1$-th rows of $\alpha$. The integers $i$ and $j$ are, collectively, the indexes of the balloon. 
Formally, we have

$$
\left(\alpha \odot_{i, j} \beta\right)_{x}= \begin{cases}\alpha_{x} & \text { if } x \leqslant i \text { and } \alpha_{x} \leqslant j \\ \alpha_{x}+|\beta| & \text { if } x \leqslant i \text { and } \alpha_{x}>j \\ \beta_{x-i}+j & \text { if } x>i \text { and } x \leqslant i+|\beta| \\ \alpha_{x-|\beta|} & \text { if } x>i+|\beta| \text { and } \alpha_{x-|\beta|} \leqslant j \\ \alpha_{x-|\beta|}+|\beta| & \text { if } x>i+|\beta| \text { and } \alpha_{x-|\beta|}>j\end{cases}
$$

As before, the balloon notation is not associative. Unlike 2413-balloons, which have to be interpreted as right-associative, generalized balloons can use brackets to define associativity. Note that the 2413-balloon defined in Section 2 are written as $2413 \odot_{2,2} \beta$ in our generalized notation.

We remark that for any $\alpha$ and any $\beta$, we have $\alpha \odot_{0,0} \beta=\alpha \oplus \beta$, and we can easily determine $\mu[\alpha \oplus \beta]$ using results from Propositions 1 and 2 of Burstein, Jelínek, Jelínková and Steingrímsson [4].

\subsection{Generalised 2413-balloons}

If we restrict $\alpha$ to 2413 , then, up to symmetry, there are seven possible values for the indexes: $(0,0),(0,1),(0,2),(1,0),(1,1),(1,2)$, and $(2,2)$. Theorem 16 handles the case where the indexes are $(2,2)$, and [4] handles the case where the indexes are $(0,0)$. For the other indexes, we have

Conjecture 17. Let $\pi=2413 \odot_{i, j} \beta$, where $(i, j) \in\{(0,1),(0,2),(1,1),(1,2)\}$. Then

$$
\mu[\pi]= \begin{cases}0 & \text { If }(i, j)=(0,1) \text { and } \beta=\tau \oplus 1 \\ 0 & \text { If }(i, j)=(0,2) \text { and } \beta=\tau \ominus 1 \\ 0 & \text { If }(i, j)=(1,1) \text { and } \beta=1 \ominus \tau \text { or } 12 \\ 0 & \text { If }(i, j)=(1,2) \text { and } \beta=1 \oplus \tau \\ \mu[\beta] & \text { Otherwise. }\end{cases}
$$

and

Conjecture 18. Let $\pi=2413 \odot_{1,0} \beta$. Then

$$
\mu[\pi]= \begin{cases}6 & \text { If } \beta=1 \\ -2 & \text { If } \beta=21 \\ 0 & \text { If } \beta=312 \\ 2 \mu[\beta] & \text { If } \beta=2413 \odot_{1,0} \gamma \\ \mu[\beta] & \text { Otherwise. }\end{cases}
$$


We remark here that Theorem 16 and Conjecture 18 have a very similar structure. It is not clear to us whether this similarity is coincidental, or whether there is some deeper reason.

\subsection{Bounding the Möbius function on hereditary classes}

Corollary 24 in Burstein, Jelínek, Jelínková and Steingrímsson [4] gives us that if $\pi$ is separable, then $\mu[\pi] \in\{0, \pm 1\}$. The simple permutations in the hereditary class of separable permutations are 1, 12 , and 21 . In Remark 14 we have unbounded growth where the simple permutations in the hereditary class are just 1, 12, 21, 2413, and 25314, so adding 2413 and 25314 to the simple permutations moves us from bounded growth to unbounded growth. This then leads to:

Question 19. If $C$ is a hereditary class containing just the simples 1, 12, 21 and 2413, and $\pi \in C$, then is $\mu[\pi]$ bounded? Further, if $D$ is a hereditary class containing just the simples $1,12,21,2413$, and 3142 , and $\pi \in D$, then is $\mu[\pi]$ bounded?

Acknowledgements. I would like to thank my supervisor, Robert Brignall, for his patience and help while I was writing this paper, and Einar Steingrímsson and Jan Kynčl for their comments. I would also like to thank Jan for spotting an error in an earlier version of what is now Lemma 10, and an anonymous reviewer for identifying an error in Lemma 15, and suggesting a solution.

\section{References}

[1] M. H. Albert and M. D. Atkinson. Simple permutations and pattern restricted permutations. Discrete Mathematics, 300(1):1-15, 2005.

[2] R. Brignall, V. Jelínek, J. Kynčl, and D. Marchant. Zeros of the Möbius function of permutations. Mathematika, 65(4):1074-1092, 2019.

[3] R. Brignall and D. Marchant. The Möbius function of permutations with an indecomposable lower bound. Discrete Mathematics, 341(5):1380-1391, 2018.

[4] A. Burstein, V. Jelínek, E. Jelínková, and E. Steingrímsson. The Möbius function of separable and decomposable permutations. Journal of Combinatorial Theory, Series A, 118(8):2346-2364, 2011.

[5] V. Jelínek, I. Kantor, J. Kynčl, and M. Tancer. On the growth of the Möbius function of permutations. Journal of Combinatorial Theory, Series A, 169:105-121, 2020.

[6] J. P. Smith. On the Möbius function of permutations with one descent. Electronic Journal of Combinatorics, 21(2):\#P2.11, 2014.

[7] R. P. Stanley. Enumerative Combinatorics, Volume 1. Cambridge University Press, New York, 2012.

[8] H. S. Wilf. The patterns of permutations. Discrete Mathematics, 257(2):575-583, 2002. 